\title{
A Fast Image Compression Algorithm Based on Wavelet Transform
}

\author{
Xiangjun $\mathrm{Li}^{\mathbf{1 , 2}}$ \\ ${ }^{1}$ College of Physics and Electronic Information, Yan'an University, Yan'an 716000, Shaanxi, China \\ ${ }^{2}$ Shaanxi Key Laboratory of Intelligent Processing for Big Energy Data, Yan'an University, Yan'an \\ 716000, Shaanxi, China \\ Shuili Zhang ${ }^{1,2}$ \\ ${ }^{1}$ College of Physics and Electronic Information, Yan'an University, Yan'an 716000, Shaanxi, China \\ ${ }^{2}$ Shaanxi Key Laboratory of Intelligent Processing for Big Energy Data, Yan'an University, Yan'an \\ 716000, Shaanxi, China \\ Haibo Zhao \\ ${ }^{1}$ College of Physics and Electronic Information, Yan'an University, Yan'an 716000, Shaanxi, China \\ ${ }^{2}$ Shaanxi Key Laboratory of Intelligent Processing for Big Energy Data, Yan'an University, Yan'an \\ 716000, Shaanxi, China
}

Received: February 21, 2021. Revised: June 27, 2021. Accepted: July 28, 2021. Published: July 30, 2021.

\begin{abstract}
With multimedia becoming widely popular, the conflict between mass data and finite memory devices has been continuously intensified; so, it requires more convenient, efficient and high-quality transmission and storage technology and meanwhile, this is also the researchers' pursuit for highly efficient compression technology and it is the fast image transmission that is what people really seek. This paper mainly further studies wavelet analysis and fractal compression coding, proposes a fast image compression coding method based on wavelet transform and fractal theory, and provides the theoretical basis and specific operational approaches for the algorithm. It makes use of the smoothness of wavelet, the high compression ratio of fractal compression coding and the high quality of reconstructed image. It firstly processes the image through wavelet transform. Then it introduces fractal features and classifies the image according to the features of image sub-blocks. Each class selects the proper features. In this way, for any sub-block, it only needs to search the best-matched block in a certain class according to the corresponding features. With this method, it can effectively narrow the search in order to speed up coding and build the relation of inequality between the sub-block and the matching mean square error. So, it can effectively combine wavelet transform with fractal theory and further improves the quality of reconstructed image. By comparing the simulation experiment, it
\end{abstract}

objectively analyzes the performance of algorithm and proves that the proposed algorithm has higher efficiency.

Keywords - Image Compression; Fractal Theory; Sub-Block Feature; Self-Similarity; Wavelet Transform.

\section{INTRODUCTION}

On the one hand, the development of fractal coding has strongly promoted the development of image coding technology, but the compression time is still long and can't meet the requirements of real-time processing. On the other hand, the wavelet coefficients of the image are obtained after wavelet transform. Although the amount of data before and after wavelet transform is the same, after wavelet transform, the energy of the image is redistributed. The low-frequency sub-bands concentrates most of the energy, and the three high-frequency sub-bands have less energy. As for the low-frequency sub-bands, more quantization levels are used as much as possible. In recent years, the combination of fractal coding and wavelet coding has achieved great success. After multi-level wavelet decomposition, the image has strong similarity between different sub-bands, and the advantage of fractal lies in the image with strong self-similarity. With the rapid development of information age, an image contains massive, intuitive and understandable information and it is the principal element of multi-media information. People have 
been exchanging multi-media information more and more frequently, especially in image information and they have higher and higher requirements for the speed and quality of image exchange. These rapidly increased image data have put great pressure on cost and quality maintenance in both the bandwidth-limited transmission and file storage [1]. For the existing multi-media applications, these uncompressed digital images have such a tremendous scale of data that it is usually unrealistic or inefficient to directly transmit or store image information without processing and that it is a bottleneck for people to process image data highly efficiently with the current technical conditions. Image coding mainly studies the compression rate of digital images. In early days, the research of image compression is only confined to still images [2]. Since 1980, along with the swift development of digital signal processing, computer science, multi-media technology and digital communications, the research has been expanded to moving images. Some international organizations have successively defined some algorithms and compression standards and further promoted image data processing and coding compression [3]. Fractal theory has such geometric features as self-similarity and self-affinity, solves the difficulty of mathematical description and provides an effective method to study non-linear science. And fractal image compression coding is one of the best ways to determine image compression. Although fractal image compression coding methods have high compression ratios and fast decoding, but the coding computation is complex and the coding time is long; therefore, it needs to continue to study new and improved fractal coding [4]. The wavelet image generated from wavelet transform has the quantity of data equal to that of the original image; in another word, wavelet transform itself doesn't have the function of compression. The reason why it is used in image compression is that the new wavelet image has different characteristics from the original image, as shown in the fact that the image energy is mainly concentrated on the low-frequency part with less in the horizontal, vertical and diagonal parts [5]. Wavelet analysis is a time-frequency analysis of signal. It has the characteristic of multi-resolution analysis and the ability to represent the local energy of signal. As a time-frequency local analysis with a fixed window size (area) but changeable shape, wavelet transform is also known as a "mathematical microscope" [6]. The horizontal, vertical and diagonal parts represent the edge information of the original image in the corresponding parts and they have distinct directions. With high compression ratios, fractal image compression algorithm can ensure higher quality of the reconstructed image, winning itself a place in the study of image compression [7]. But fractal image coding requires to search the best-matched block among massive codebooks, which takes a plenty of time and which goes against the promotion and applications of fractal image compression coding. Worse still, there will be "block artifact" in the coding process, which also affects the quality of the reconstructed image [8]. Therefore, besides accelerating the coding speed, to improve the reconstructed image quality is also a key research direction in this paper.

Wavelet analysis can be traced back to Haar's small wave orthonormal basis in 1910. In 1987, Mallat skillfully introduced the idea of multi-scale analysis in the field of computer vision into the construction of wavelet function and the decomposition and reconstruction of signal according to wavelet transform, successfully unifying the construction of specific wavelet function before. The discretization of wavelet transform is studied, and Mallat algorithm is applied to image decomposition and reconstruction [9]. In 1996, the traditional filter banks were extended to vector filter banks and block filter banks. The theoretical system of vector filter banks was initially formed, and its relationship with multiwavelet transform was established [10]. The object of vector filter bank is vector signal. It can remove the correlation between vectors and keep the correlation within vectors, so it is more suitable for vector quantization. As a vector transformation, it is very effective in image compression [11]. Compression can be divided into two categories: the first type of compression process is reversible, that is to say, the original image can be completely recovered from the compressed image without any loss of information, which is called lossless compression; the second type of compression process is irreversible, unable to completely recover the original image, with some loss of information, which is called lossy compression [12]. Although we hope to achieve lossless compression, the compression ratio of lossy compression is usually higher than that of lossless compression. The traditional first generation image compression coding method is proposed to solve the problem of data redundancy caused by image linear similarity, and has made many achievements, such as JPEG [13]. With the progress of the times, the shortcomings of the first generation of image compression methods are more and more obvious, such as the serious block effect will appear when obtaining a higher compression ratio. In order to overcome these shortcomings, a new generation of image coding method was proposed in 1985 , trying to combine the human visual and sensory characteristics with the image source characteristics, in order to improve the image compression ratio. In multimedia applications, common compression methods include PCM (pulse code modulation), predictive coding, transform coding, interpolation and extrapolation, statistical coding, vector quantization and subband coding. Fractal image compression was first proposed by Barnsley in 1988 [14]. He observed that the image created by the iterated function system has a natural appearance, and deduced that the natural image can also be represented by the iterated function system. In 1992, Jacquin proposed an improved algorithm called basic fractal image compression [15]. Jacquin's scheme uses partition iterated function system to find affine transformation information automatically without human-computer interaction, and its application scope is also extended to general natural images. Since then, fractal image coding has become a research hotspot. Compared with other mainstream image compression algorithms, basic fractal coding has the advantages of high compression ratio, excellent reconstructed image quality and decoding independent of image resolution [16]. At the same time, it brings new problems. The large-scale computation of local compressed affine transform information leads to the long encoding time, which limits the application of fractal coding. Generally speaking, the research focus and direction of fractal coding image compression are mainly reflected in improving the codebook search time, the selection of compression mapping mode, the quantization of image parameters, reducing the complexity of fractal coding decoding algorithm, and so on 
[17]. Many researchers provide a large number of improvement schemes to make up for the shortcomings of fractal coding, mainly by improving the compression ratio, improving the image quality after decoding and reducing the time complexity of the algorithm. From the effect of fractal image compression technology at this stage, there are still many shortcomings [18].

This paper has first introduced the basic principle, evaluation criteria and current development of image compression and then raised improved method to address the problems in image compression coding. First of all, it decomposes the image with wavelet transform according to the characteristic that wavelet transform can change the distribution of energy and then it has integrated fractal features, achieved the hybrid coding combined with wavelet transform and fractal theory and improved the clarity of the reconstructed image. On the basis of global search fractal coding algorithm, it has used the self-similarity of image sub-block, and come up with an improved algorithm to narrow down the search space according to its relation with the matching mean square error. This improved algorithm can convert the global search for the best-matched block into local search so as to shorten the coding time and further improve the image of reconstructed image.

\section{IMAGe Coding AND DECODING}

\section{A. Image redundancy}

Coding redundancy is to achieve data compression by using as few bits as possible to express as many gray levels as possible. This kind of processing is usually called variable length coding. If the number of coding symbols used in encoding the gray level of an image is more than the actual number of symbols needed to represent each gray level, the image obtained by this encoding method contains coding redundancy. Usually, if the encoding (such as gray level value) assigned to the event set does not make full use of the probability of various results to select, there will be coding redundancy. When the gray level of an image is directly represented by natural binary coding, redundancy always exists. In this case, the fundamental basis of dealing with coding redundancy is that the image is composed of regular objects with predictable shape and reflection to some extent, and usually the image is sampled so that the objects described are much larger than the image elements. In most images, the normal result is that one gray level is more likely to appear than other gray levels (that is, the histogram of most images is uneven). The natural binary coding of their gray level allocates the same number of bits to the value with maximum possibility and minimum possibility, so the minimum value cannot be obtained, resulting in coding redundancy [19] [20].

There are a lot of redundant data in image data. In general, there may be the following redundancy in image data:

(1) Spatial redundancy (inter pixel redundancy, geometric redundancy): This is a kind of redundancy that often exists in image data. In the same image, the surface physical properties of regular objects and regular background (rule means that the surface is orderly rather than completely disordered arrangement) are correlated. The optical imaging results of these correlations are shown as data redundancy in the digital image.
(2) Time redundancy: in the sequence image (TV image, moving image), there is a large correlation between two adjacent frames.

(3) Information entropy redundancy: also known as coding redundancy. If the average number of bits used by each pixel in the image is greater than the information entropy of the image, there is redundancy in the image, which is called information entropy redundancy.

(4) Structural redundancy: some images have strong texture structure, such as wallpaper, straw mat and other images, which is called structural redundancy.

(5) Knowledge redundancy: the understanding of many images is quite related to some basic knowledge. For example, the face image has a fixed structure, such as the nose above the mouth, the eyes above the nose, and the nose is located on the middle line of the face image. This kind of regular structure can be obtained from prior knowledge and background knowledge. This kind of redundancy is called knowledge redundancy.

(6) Psychological visual redundancy: the attention of the human visual system to the image field is non-uniform and non-linear, especially the visual system is not able to perceive any changes in the image field, that is, the eye does not have the same sensitivity to all information, some information is not so important compared with other information in the normal visual perception process It can be considered as psychological and visual redundancy, and removing this information will not significantly reduce the quality of the image.

From the perspective of information theory, the data used to describe the image source is composed of effective information and redundant data. Removing redundant data can save data in storage and transmission without damaging the effective information of image source. In some cases, distortion is allowed to a certain extent, such as the limitation of human eyes on image gray resolution, the limitation of monitor display resolution and so on. However, these distortions do not hinder the practical application of the image, which can also be used to compress the image source to a certain extent.

\section{B. Lossless image compression}

Lossless image compression is based on the statistical redundancy of data, which can recover the original data completely without any distortion. This kind of method is widely used in the compression of text data, programs and image data in special applications. The commonly used lossless compression methods include Shannon coding, Huffman coding, run length coding, LZW (Lempel Ziv Welch) coding and arithmetic coding.

The simplest method of lossless image compression is to reduce the only coding redundancy, which usually exists in the natural binary coding process of image gray level. In this way, a variable length coding structure is needed, which can assign the shortest codeword to the gray level with the largest probability of occurrence. Here, several best and near best coding techniques are analyzed for constructing such codewords. These technologies are expressed in the language of information theory. In fact, the source symbol may be either the gray level of the image or the output of the gray level mapping operation (pixel difference, stroke width, etc.). Huffman coding is a typical lossless compression method [21]. 
(1) Firstly, the probability of each symbol appearing in the source is counted, and the order is from large to small;

(2) The minimum two probabilities are added and combined into a new probability, and the remaining probabilities form a new probability set;

(3) The new probability set is reordered, and the two minimum probabilities are added again to form a new probability set, which is repeated until the sum of the last two probabilities is 1 ;

(4) Allocate codewords: codeword allocation starts from the last step in reverse. For the two probabilities of each addition, assign " 0 " to the large one and " 1 " to the small one. When reading out, it starts from the symbol and goes to the last probability and " 1 ", and arranges the " 0 " and " 1 " encountered on the route from the lowest to the highest order, which is the Hoffman coding of the symbol.

Huffman coding is not unique. Huffman coding has different coding efficiency for different sources. The results of Huffman coding are not equal in length, so it is very difficult to implement hardware, and the error propagation is serious. In general, the efficiency of Huffman coding is higher than other coding algorithms, and it is the best variable length code. However, Huffman coding depends on the statistical characteristics of the source, so the probability characteristics of the source must be calculated before coding, which limits the practical application of Huffman coding [22].

\section{Lossy image compression coding}

The so-called lossy compression makes use of humans not being sensitive to certain frequency components in the image or sound wave and it allows the loss of certain information in the compression. Although the application of lossy compression can't completely restore the original data, the decreased influenced of the lossy part on comprehending the original image can result in much greater compression ratio. Lossy compression is widely applied in the compression of audio, image and video data.

1) Differential Pulse Code Modulation (DPCM) Coding

DPCM coding is a kind of linear predictive coding (LPC) and it uses the past sampled value to predict the current sampled value and then code their difference value. Differential coding can enhance the coding frequency. And this technology has been used to simulate digital communications of signals. For some signals (e.g. image signal), because they have bigger instantaneous slopes, it is very easy for them to cause over-loading; therefore, simple delta modulation can't be used for coding and besides, such kind of signals don't have syllable characteristic like audio signal, so syllable companding can't be adopted and only instantaneous companding can be used. So for this kind of signals with larger instantaneous slopes, a modulation method which integrates delta modulation and pulse code modulation, which is called pulse code delta modulation or differential pulse code modulation and represented by Differential Pulse Code Modulation (DPCM) [23].

Assume that the discrete time analog signal is set $\left\{X_{K}\right\}$ and the signal value at moment $K$ is $X_{K}$. If prediction is made with the linear combination of the past $N$ signals, then the predictive value is

$$
\left\{\hat{X}_{K}\right\}=\sum_{i=1}^{N} a_{i} X_{K-1}
$$

There is a signal difference $e_{K}$ between the actual value $X_{K}$ and the predictive value $\hat{X}_{K}$, i.e.

$$
e_{K}=X_{K}-\hat{X}_{K}=X_{K}-\sum_{i=1}^{N} a_{i} \hat{X}_{K-1}
$$

If proper $N$ and $a_{i}$ are chosen, the characteristic of $e_{K}$ becomes the white-noise process with a mean of 0 and it is marked as $W_{K}$. The obviously recovered $X_{K}$ is

$$
X_{K}=\sum_{i=1}^{N} a_{i} X_{K-i}+W_{K}
$$

\section{2) Discrete Cosine Transform Coding}

Discrete Cosine Transform (DCT) is a transform coding method frequently used in digital rate compression. The Fourier transform of any continuous real symmetrical function only includes cosine item; so cosine transform also has definite physical meaning, just as Fourier transform [24].

(1) $1 \mathrm{D}-\mathrm{DCT}$

Assume that $\{X(m) \mid m=0,1, \cdots, N-1\}$ is the data series obtained from sampling bandwidth-limited signal $x(t)$ with $N$ sampled values and its 1D-DCT is defined as below.

$$
\begin{aligned}
Y(u) & =C(u) \sqrt{\frac{2}{N}} \sum_{m=0}^{N-1} X(m) \cos \frac{(2 m+1) u \pi}{2 N}, \\
u & =1,2, \cdots, N-1
\end{aligned}
$$

$$
\text { Where } C(u)= \begin{cases}1 / \sqrt{2} & (u=0) \\ 1 & (\text { Others })\end{cases}
$$

The inverse transform of 1D-DCT (1D-IDCT) is defined as follows:

$$
\begin{aligned}
& X(m)=\sqrt{\frac{2}{N}} \sum_{m=0}^{N-1} C(u) Y(u) \cos \frac{(2 m+1) u \pi}{2 N}, \\
& m=1,2, \cdots, N-1
\end{aligned}
$$

Both of their transform kernel is

$$
\begin{aligned}
& a(u, m)=C(u) \sqrt{\frac{2}{N}} \cos \frac{(2 m+1) u \pi}{2 N}, \\
& \{a(u, m) \mid u=0,1, \cdots, N-1\}
\end{aligned}
$$

(2) Principles of 2D-DCT

The definition of 1D-DCT can be expanded to 2D-DCT. Assume that $\{X(m, n) \mid m=0,1, \cdots, M-1 ; n=0,1, \cdots, N-1\}$ is 
the data matrix of $2 \mathrm{D}$ image signals and the $2 \mathrm{D}-\mathrm{DCT}$ is defined as below:

$$
\begin{aligned}
& Y(u, v)=\frac{2}{\sqrt{M N}} C(u) C(v) \sum_{m=0}^{M-1} \sum_{n=0}^{N-1} X(m, n) \\
& \cos \frac{(2 m+1) u \pi}{2 M} \cos \frac{(2 n+1) v \pi}{2 N}
\end{aligned}
$$

Where $u=0,1, \cdots, M-1 ; v=0,1, \cdots, N-1$.

$$
C(u), C(v)= \begin{cases}1 / \sqrt{2} & (u, v=0) \\ 1 & (\text { Others })\end{cases}
$$

Its inverse transform, 2D-IDCT is defined as:

$$
\begin{aligned}
& X(m, n)=\frac{2}{\sqrt{M N}} \sum_{u=0}^{M-1} \sum_{v=0}^{N-1} C(u) C(v) Y(u, v) \\
& \cos \frac{(2 m+1) u \pi}{2 M} \cos \frac{(2 n+1) v \pi}{2 N}
\end{aligned}
$$

Where $m=0,1, \cdots, M-1 ; n=0,1, \cdots, N-1$; Separate the transform kernel and get 2 1D-DCT.

$$
\begin{aligned}
& a(u, v, m, n)=a_{1}(u, m) a_{2}(v, n)= \\
& C(u) \sqrt{\frac{2}{M}} \cos \frac{(2 m+1) u \pi}{2 M} C(v) \sqrt{\frac{2}{N}} \cos \frac{(2 n+1) v \pi}{2 N}
\end{aligned}
$$

(3) DCT in Image Compression Coding

It conducts video compression coding with DCT. At first, it decomposes the image into $8 * 8$ blocks. Then it performs $2 \mathrm{D}$ discrete transform on each block and transforms every of them into 64 DCT coefficient values. At last, it codes and transmits the DCT coefficients, performs 2D-IDCT to every block during the decoding and combines the blocks after the inverse transform into an image.

Therefore, 2D-DCT can be materialized as below:

$$
\begin{aligned}
& Y(u, v)=\frac{1}{4} C(u) C(v) \sum_{m=0}^{7} \sum_{n=0}^{7} X(m, n) \\
& \cos \frac{(2 m+1) u \pi}{16} \cos \frac{(2 n+1) v \pi}{16}
\end{aligned}
$$

Where $u=0,1, \cdots, 7, v=0,1, \cdots, 7$. In other words, it has transformed $8 * 8$ 2D-DCT into $2 N=81 \mathrm{D}$-DCT.

DCT is a real orthogonal transformation and the primary function of its transform kernel orthogonalizes. Sequence DCT is the symmetric expansion form of discrete Fourier transform (DFT) with kernel to be separated and it can be replaced by 2 1D transform. The energy of cosine transform has the trend to focus on low frequency. Cosine transform has fast transform and just like Fourier transform, it works differently on odd and even [25].
III. 2D IMAGE WAVELET TRANSFORM AND FASt ALGORITHM

In order to process $2 \mathrm{D}$ image signals, $2 \mathrm{D}$ wavelet and $2 \mathrm{D}$ wavelet transform must be introduced. There are 2 kinds of 2D multi-resolution analysis: one is separable and the other is inseparable. $L^{2}\left(R^{2}\right)$ is used to represent the space of square integrable function on the place, namely

$$
\left.f(x, y) \in L^{2}\left(R^{2}\right) \Leftrightarrow \int_{-\infty}^{+\infty} \int_{-\infty}^{+\infty} f(x, y)\right|^{2} d x d y<\infty
$$

An image in the finite region on the place has limited energy. Assume that $f(x, y)$ is an image, the area of the region enclosed by its domain of definition is $\mathrm{D}$ and the maximum brightness value of $f(x, y)$ is $\mathrm{M}$, namely $f(x, y) \leq M$, then

$$
\left.\int_{-\infty}^{+\infty} \int_{-\infty}^{+\infty} f(x, y)\right|^{2} d x d y \leq M^{2} D<+\infty
$$

After introducing the inner product of $L^{2}\left(R^{2}\right)$ space,

$$
\langle f, g\rangle=\int_{R^{2}} f(x, y) \overline{g(x, y)} d x d y, f, g \in L^{2}\left(R^{2}\right)
$$

The corresponding bound norm is defined as

$$
\|f\|_{L^{2}\left(R^{2}\right)}=\langle f, f\rangle^{1 / 2}, f \in L^{2}\left(R^{2}\right)
$$

In the event of no confusion, bound norm is also marked as $\|f\|_{L^{2}}$. The Fourier transform of $f(x, y)$ is defined as

$$
\hat{f}(\zeta)=\hat{f}\left(\zeta_{1}, \zeta_{2}\right)=\int_{R^{2}} f(x, y) e^{-i\left(x \zeta_{1}+y \zeta_{2}\right)} d x d y
$$

Assume that $P$ and $D$ are two linear spaces with finite dimensions or countably infinite dimensions and the bases of $\mathrm{F}$ and $D$ are $\ldots, f_{-1}, f_{0}, f_{1} \ldots$ and $\ldots, d_{1}, d_{0}, d_{1} \ldots$ respectively. The space with the elements in the form of $f_{i} d_{i}(i=0, \pm 1, \pm 2, \cdots ; j=0, \pm 1, \pm 2, \cdots)$ as the base is defined as $H$, which is the tensor product space of $F$ and $D$, as represented as $H=F \otimes D$

If both $\mathrm{F}$ and $D$ are function spaces and $x$ and $y$ are the independent variables of $\mathrm{F}$ and $D$ respectively, then the elements in the tensor product space $H$ is called 2D tensor product function or tensor product surface.

Now, assume that $\left\{V_{k}^{1}\right\}$ and $\left\{V_{k}^{2}\right\}$ are 2 multi-resolution analyses generated by scale functions $\phi^{1}(x)$ and $\phi^{2}(y)$ respectively, then the tensor product spaces of $V_{k}^{1}$ and $V_{k}^{2}$ can be obtained:

$$
V_{k}=V_{k}^{1} \otimes V_{k}^{2}
$$

As the base of $V_{k}^{1}$ is $\left\{2^{k / 2} \phi^{1}\left(2^{k} x-j\right)\right\}$ and that of $V_{k}^{2}$ is $\left\{2^{k / 2} \phi^{2}\left(2^{k} y-l\right)\right\}$, the base of $V_{k}$ is $\left\{2^{k} \phi^{1}\left(2^{k} x-j\right) \phi^{2}\left(2^{k} y-l\right)\right\}$.

For the two-variable function $f(x, y)$, mark is introduced:

$$
f_{k ; j, i}(x, y)=2^{k} f\left(2^{k} x-j, 2^{k} y-l\right)
$$


i.e. $\phi(x, y)=\phi^{1}(x) \phi^{2}(y)$

Then $\left\{\phi_{k ; j, i}(x, y): j, l \in Z\right\}$ is the base of $V_{k}$. In this way, $\left\{V_{k}\right\}$ forms one multi-resolution analysis in $L^{2}\left(R^{2}\right)$ and $\phi(x, y)$ is the corresponding scale function.

Assume that the complementary space of $V_{k}^{1}$ about $V_{k+1}^{1}$ is $W_{k}^{1}$ and that of $V_{k}^{2}$ about $V_{k+1}^{2}$ is $W_{k}^{2}$, namely

$V_{k+1}^{1}=V_{k}^{1}+W_{k}^{1}, V_{k+1}^{2}=V_{k}^{2}+W_{k}^{2}$

Now, assume that $\psi^{1}(x)$ generates $W_{0}^{1}$ and $\psi^{2}(x)$ generates $W_{0}^{2}$, namely

$$
\begin{aligned}
& W_{0}^{1}:=\operatorname{clos}_{L^{2}(R)}\left\langle\psi^{1}(x-k): k \in Z\right\rangle \\
& W_{0}^{2}:=\operatorname{clos}_{L^{2}(R)}\left\langle\psi^{2}(x-k): k \in Z\right\rangle
\end{aligned}
$$

At that time,

$$
\begin{aligned}
V_{k+1} & =V_{k+1}^{1} \otimes V_{k+1}^{2}=\left(V_{k}^{1}+W_{k}^{1}\right) \otimes\left(V_{k}^{2}+W_{k}^{2}\right) \\
& =V_{k}^{1} \otimes V_{k}^{2}+V_{k}^{1} \otimes W_{k}^{2}+W_{k}^{1} \times V_{k}^{2}+W_{k}^{1} \otimes W_{k}^{2} \\
& =V_{k}+W_{k}
\end{aligned}
$$

Where

$$
\begin{aligned}
& W_{k}=W_{k}^{(1)}+W_{k}^{(2)}+W_{k}^{(3)}, \\
& W_{k}^{(1)}=V_{k}^{1} \otimes W_{k}^{2}, W_{k}^{(2)}=W_{k}^{1} \otimes V_{k}^{2}, W_{k}^{(3)}=W_{k}^{1} \otimes W_{k}^{2}
\end{aligned}
$$

Likewise, as the base of $V_{k}^{1}$ is $\left\{2^{k / 2} \phi^{1}\left(2^{k} x-j\right)\right\}$ and that of $W_{k}^{2}$ is $\left\{2^{k / 2} \psi^{1}\left(2^{k} y-l\right)\right\}$, then the base of $W_{k}^{(1)}$ is $\left\{2^{k} \phi^{1}\left(2^{k} x-j\right) \psi^{2}\left(2^{k} y-l\right)\right\}$, marked as

$$
\psi^{1}(x, y)=\phi^{1}(x) \psi^{2}(y)
$$

Then the base of $W_{k}^{(1)}$ is $\left\{\psi_{k, j, i}^{1}: j, l \in Z\right\}$ and likewise, it is marked as

$$
\begin{aligned}
& \psi^{2}(x, y)=\psi^{1}(x) \phi^{2}(y) \\
& \psi^{3}(x, y)=\psi^{1}(x) \psi^{2}(y)
\end{aligned}
$$

The base of $W_{k}^{(1)}$ is $\left\{\psi_{k ; j, i}^{2}: j, l \in Z\right\}$ and that of $W_{k}^{(3)}$ is $\left\{\psi_{k ; j, i}^{3}: j, l \in Z\right\}$.

It can be seen that different from the fact that $1 \mathrm{D}$ only has 1 scale function and 1 wavelet function, 2D has 1 scale function $\phi(x, y)$ and 3 wavelet functions $\psi^{1}(x, y), \psi^{2}(x, y), \psi^{3}(x, y)$. (21):

Similar to 1D, there are following solutions to Formula

$$
L^{2}\left(R^{2}\right)=\cdots+W_{-1}+W_{0}++W_{1}+\cdots
$$

For $\forall f(x, y) \in L^{2}\left(R^{2}\right)$, there is only 1 solution

$$
f(x, y)=\cdots+d_{-1}(x, y)+d_{0}(x, y)+d_{1}(x, y)+\cdots
$$

Where $d_{k}(x, y) \in W_{k}$.

If $\phi^{1}(x), \phi^{2}(y)$ and $\psi^{1}(x), \psi^{2}(y)$ are semi-orthogonal scaling function and semi-orthogonal wavelet function, the above direct decomposition can be turned into orthogonal decomposition:

$$
L^{2}\left(R^{2}\right)=\cdots \oplus W_{-1} \oplus W_{0} \oplus W_{1} \oplus \cdots
$$

At this time $W_{k} \perp W_{n}, k \neq n$, namely $\left\langle d_{k}, d_{n}\right\rangle=0, k \neq n$, where $d_{k} \in W_{k}, d_{n} \in W_{n}$.

Assume that $f_{k}(x, y) \in V_{k}, d_{k}(x, y) \in W_{k} \quad, \quad$ then $f_{k+1}(x, y)=f_{k}(x, y)+d_{k}(x, y)$.

For any $\mathrm{K}, f_{k} \in V_{k}, d_{k} \in W_{k}$. In this way, $d_{k} \in W_{k}$ can be further decomposed in to $d_{k}=d_{k}^{(1)}+d_{k}^{(2)}+d_{k}^{(3)}$.

Where $d_{k}^{(i)} \in W_{k}^{(i)}(i=1,2,3)$, and $f_{k+1}(x, y) \in V_{k+1}$. equation:

Make use of the 1D double-scaling equation and wavelet

$$
\begin{aligned}
& \left\{\begin{array}{l}
\phi^{1}(x)=\sum_{n} h_{n}^{1} \phi^{1}(2 x-n) \\
\psi^{1}(x)=\sum_{n} g_{n}^{1} \phi^{1}(2 x-n)
\end{array}\right. \\
& \left\{\begin{array}{l}
\phi^{2}(x)=\sum_{n} h_{n}^{2} \phi^{2}(2 x-n) \\
\psi^{2}(x)=\sum_{n} g_{n}^{2} \phi^{2}(2 x-n)
\end{array}\right.
\end{aligned}
$$

The double-scaling relation of $2 \mathrm{D}$ tensor product is

$$
\begin{gathered}
\left\{\begin{array}{l}
\phi(x, y)=\sum_{n, m} h_{n, m} \phi(2 x-n, 2 y-m) \\
\psi^{i}(x, y)=\sum_{n, m} g_{n, m}^{i} \phi(2 x-n, 2 y-m),(i=1,2,3)
\end{array}\right. \\
\text { Where }\left\{\begin{array}{l}
h_{n, m}=h_{n}^{1} h_{m}^{2}, g_{n, m}^{1}=h_{n}^{1} g_{m}^{2} \\
g_{n, m}^{2}=g_{n}^{1} h_{m}^{2}, g_{n, m}^{3}=g_{n}^{1} g_{m}^{2}
\end{array}\right.
\end{gathered}
$$

Now, assume

$$
\begin{aligned}
& f_{k}(x, y)=\sum_{k ; n, m} c_{k ; n, m} \phi\left(2^{k} x-n, 2^{k} y-m\right) \\
& g_{k}^{(i)}(x, y)=\sum_{n, m} d_{k ; n, m}^{i} \psi^{i}\left(2^{k} x-n, 2^{k} y-m\right) \\
& \text { and } \\
& f_{k+1}(x, y)=f_{k}(x, y)+g_{k}^{(1)}(x, y)+g_{k}^{(2)}(x, y)+g_{k}^{(3)}(x, y) .
\end{aligned}
$$

The belowing Fig. 1 is the wavelet transform coefficients of different scales. 


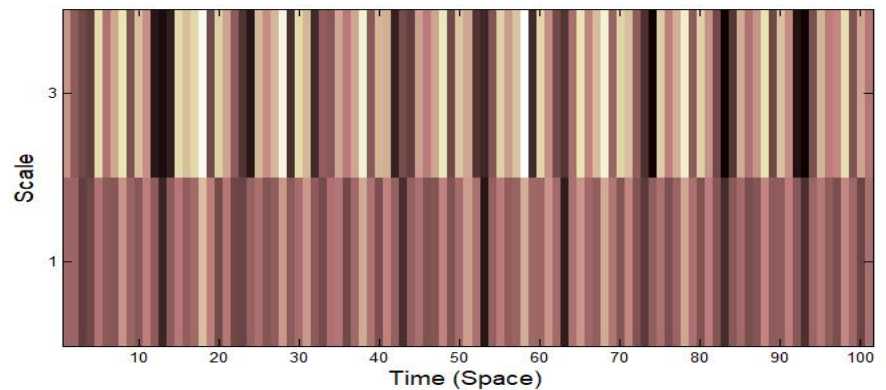

Fig 1. Wavelet transform coefficients of different scales

Then make use of the scale function $\phi(x, y)$ and 3 wavelet functions $\psi^{1}(x, y), \psi^{2}(x, y), \psi^{3}(x, y)$ as well as its orthogonality of dilation and translation and get the 2D Mallat algorithm as shown below.

(1) Decomposition algorithm

$$
\left\{\begin{array}{l}
c_{k ; n, m}=\sum_{l, j} h_{l-2 n} h_{j-2 m} c_{k+1 ; l, j} \\
d_{k ; n, m}^{1}=\sum_{l, j} h_{l-2 n} g_{j-2 m} c_{k+1 ; l, j} \\
d_{k ; n, m}^{2}=\sum_{l, j} g_{l-2 n} h_{j-2 m} c_{k+1 ; l, j} \\
d_{k ; n, m}^{3}=\sum_{l, j} g_{l-2 n} g_{j-2 m} c_{k+1 ; l, j}
\end{array}\right.
$$

(2) Reconstruction algorithm

$$
\begin{aligned}
& c_{k+1 ; n, m}=\sum_{l, j} h_{n-2 l} h_{m-2 j} c_{k ; n, m}+\sum_{l, j} h_{n-2 l} h_{m-2 j} d_{k ; n, m}^{1}+ \\
& \sum_{l, j} g_{n-2 l} h_{m-2 j} d_{k ; n, m}^{2}+\sum_{l, j} g_{n-2 l} g_{m-2 j} d_{k ; n, m}^{3}
\end{aligned}
$$

In the same way, 1D bi-orthogonal multi-resolution analysis can be used to get 2D bi-orthogonal multi-resolution analysis. As long as the corresponding decomposition and reconstruction filters are replaced, the Mallat algorithm of 2D bi-orthogonal multi-resolution analysis can be obtained. The sequence $\left\{c^{k}, d_{k}^{1}, d_{k}^{2}, d_{k}^{3}\right\}$ is called as the primary 2D wavelet transform of $c^{k+1}$.

With the above analysis, the methods can be analyzed to process 2D discrete image signals. Assume that $\left\{b_{n, m}\right\}(n=0,1, \cdots, N-1)$ is an input image and the distance between its pixels is $N^{-1}$ and $N=2^{L}$. Then an approximation function $f(x, y)=\sum_{n, m} c_{n, m}^{L} \phi_{L, n, m}(x, y) \in V_{L}^{2}$ of $b_{n, m}$ and scale $2^{\mathrm{L}}$ can be connected. In it, $c_{n, m}^{L}=\left\langle f, \tilde{\varphi}_{L, n, m}\right\rangle, \phi, \tilde{\phi}$ are 2 dual scaling functions, making $b_{n, m}$ the uniform sampling of $f(x, y)$, namely $b_{n, m}=f\left(N^{-1} n, N^{-1} m\right)$. Besides, according to $c_{n, m}^{L}=\left\langle f, \tilde{\phi}_{L, n, m}\right\rangle$, then

$$
N c_{n, m}^{L}=\int_{-\infty}^{+\infty} \int_{-\infty}^{+\infty} f(u, v) \frac{1}{N^{-2}} \tilde{\phi}\left(\frac{u-N^{-1} n}{N^{-1}}, \frac{u-N^{-1} m}{N^{-1}}\right) d u d v
$$

As $\int_{-\infty}^{+\infty} \int_{-\infty}^{+\infty} \phi(u, v) d u d v=1$, then

$$
\int_{-\infty}^{+\infty} \int_{-\infty}^{+\infty} \frac{1}{N^{-2}} \tilde{\phi}\left(\frac{u-N^{-1} n}{N^{-1}}, \frac{u-N^{-1} m}{N^{-1}}\right) d u d v=1
$$

And $N c_{n, m}^{L}$ is the weighted mean of $f$ in a small domain of $\left(N^{-1} n, N^{-1} m\right)$, namely

$$
N c_{n, m}^{L} \approx f\left(N^{-1} n, N^{-1} m\right)=b_{n, m}
$$

If $\left\{c_{k+1, l, j}\right\}$ is seen as a 2D image signal and $\mathrm{n}$ and mare the row subscript and column subscript respectively, the following explanation can be made to the 2D wavelet transform process: firstly, it uses analytical filters $\overline{\tilde{h}}, \overline{\tilde{g}}$ to perform wavelet transform on every $\mathrm{n}$ rows and gets the low-frequency part $\sum_{j} \tilde{h}_{j-2 m} c_{k+1 ; l . j}$ and high-frequency part $\sum_{j} \tilde{g}_{j-2 m} c_{k+1 ; l . j}$; secondly, it performs wavelet transform on every m columns of the data with the analytical filters $\overline{\tilde{h}}$ and $\overline{\tilde{g}}$ : it conducts wavelet transform on every column of $\sum_{j} \tilde{h}_{j-2 m} c_{k+1 ; l . j}$ and gets the low-frequency coefficient $\sum_{1} \tilde{h}_{l-2 n}\left(\sum_{j} \tilde{h}_{j-2 m} c_{k+1 ; n . m}\right)$, i.e. $c_{j ; n, m}$ and the high-frequency coefficient $\sum_{1} \tilde{g}_{l-2 n}\left(\sum_{j} \tilde{h}_{j-2 m} c_{k+1 ; n, m}\right)$, i.e. $d_{k ; n, m}^{1} ;$ it performs wavelet transform on every row of $\sum_{j} \tilde{g}_{j-2 m} c_{k+1 ; l . j}$ and gets the low-frequency coefficient $\sum_{1} \tilde{h}_{l-2 n}\left(\sum_{j} \tilde{g}_{j-2 m} c_{k+1 ; n . m}\right)$, namely $d_{k ; n, m}^{2} \quad$ and the high-frequency coefficient $\sum_{1} \tilde{g}_{l-2 n}\left(\sum_{j} \tilde{g}_{j-2 m} c_{k+1 ; n . m}\right)$, namely $d_{k ; n, m}^{3}$. The image after the primary wavelet decomposition is made up of 4 parts:

$$
\left[\begin{array}{ll}
\left(c_{k ; n, m}\right) & \left(d_{k ; n, m}^{1}\right) \\
\left(d_{k ; n, m}^{2}\right) & \left(d_{k ; n, m}^{3}\right)
\end{array}\right]
$$

Where each sub-image is $1 / 4$ of the size of the original image. The low-frequency signal obtained from every level of transform can be decomposed recursively and the reconstruction process can also be conducted likewise; in this way, the tower structure of 2D wavelet transform is formed. Apply high-pass and low-pass filters respectively on rows and columns. Such decomposition result is still 4 images: approximate image, horizontal detail image, vertical detail image and diagonal image. The Fig.2 is the comparison of continuous wavelet and discrete wavelet.

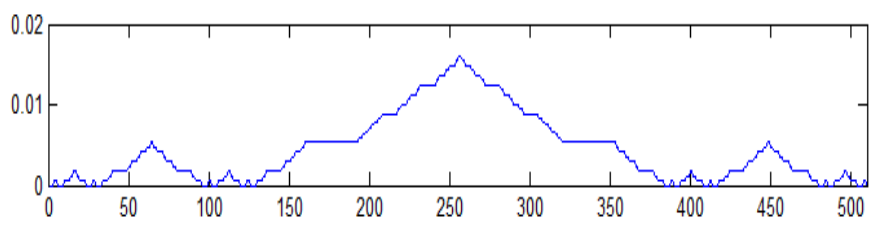

(a) Analyzed signal 


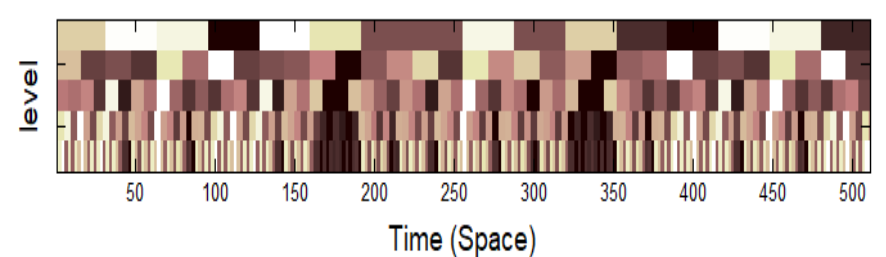

(b) Discrete Transform, absolute coefficients

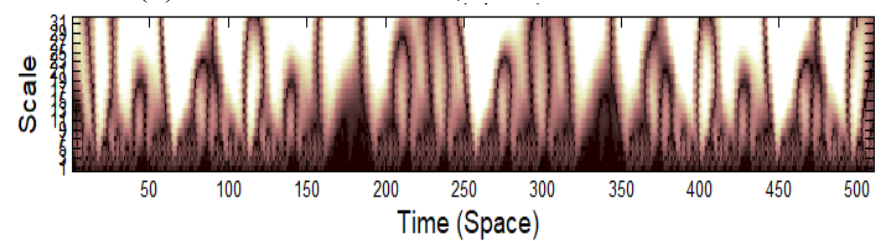

(c) Continuous Transform, absolute coefficients

Fig 2. Comparison of continuous wavelet and discrete wavelet

\section{Steps of WAVELET Fractal COMPRESSION CODING ALGORITHM}

The general steps of wavelet transform being used for image coding include: perform multi-resolution decomposition on the image, decompose it into the sub-images in different spaces and with different frequency and perform coefficient coding on sub-images. Coefficient coding is the core of using wavelet transform in compression, the essence of which is to perform quantized compression on the coefficients. Through the secondary wavelet decomposition, the image is decomposed into 7 sub-bands. Preserve the wavelet coefficients of low-frequency sub-bands and perform fractal image coding on the rest 6 . Among them, Blocks $R$ and $D$ of the secondary sub-band have become $1 / 4$ of the sizes of the corresponding primary sub-bands. follows:

The specific steps of the proposed algorithm are as

\section{(1) Coding}

(1) Wavelet decomposition: perform secondary wavelet decomposition on the original image and get 7 wavelet sub-images. Preserve the wavelet coefficient of $L L_{2}$.

(2) Generate the codebook, Take all the compression of each $D$ block as the codebook $\Omega$. Segment the image to be coded into overlapping sub-blocks with a size of $2 n \times 2 n$. For each block D, adopt the mean of 4-neighborhood pixel values and get the image blocks with a size of $n \times n$ and consider 8 isometric transformation. In this way, the set of sub-blocks constitute the codebook $\Omega$.

(3) Search the best-matched blocks. Traverse the codebook $\Omega$ for Block $R$ and search the best-matched block from them. Select the codebook block $D_{m(i)}$ which has the minimum mean square error and the isometric transform number $t$ like $R_{i}$ in the neighborhood with the initial matching blocks as the center. For all blocks $D$, first process 8 kinds of spatial transform matrix and generate 8 sub-modules of the domain of definition. Then seek the mean square error. The corresponding Block $D$ to the minimum mean square error is the best-matched block and output the fractal code of Block $R$.

(4) Search the best-matched block. For each Block R, search the best-matched block $D_{m} \in \Omega_{\eta}$ and repeat Step (3) on the rest Blocks $R$ until all Blocks $R$ are processed and output the coding obtained.

(2) Decoding

Read in the size of the original image from the coding document, construct 2 blank matrices $R$ and $D$ with the same size of the original image, reconstruct each sub-image through repeated iterations with the fractal coding of Block $\mathrm{R}$, add the corresponding wavelet coefficient symbol and perform inverse wavelet coding to get the reconstructed image. In the fractal coding, if $D$ is the best-matched block of $R$, then $E(R, D)$ will be small. There will be two circumstances and the standard deviation $\sigma_{R}$ of $R$ will be very small or the feature values of $R$ and $D$ are quite close. In order to eliminate the circumstance of $\sigma_{R}$ being small, assume a threshold $\tau>0$. When $\sigma_{R}<\tau$, Block $R$ is defined as a smooth block; otherwise, it is a non-smooth one.

By searching the secondary best solution, the quaternary group $\left(m, \hat{s}_{i}, \hat{o}_{i}, t\right)$ of fractal coding of Block $R\left(\hat{s}_{i}\right.$ and $\hat{o}_{i}$ are the quantized values of $s_{i}$ and $o_{i}$ and $t$ is the sequence of isometric transformation). In order to further accelerate the speed of the algorithm, this algorithm omits the isometric transformation step in the spatial domain fractal compression algorithm, adopts the method of encoding the positive and negative wavelet coefficients respectively, improves the compression ratio of the algorithm, and directly stores the value domain blocks with large standard deviation. The experimental results show that the improved wavelet domain search free fractal compression algorithm improves the compression ratio to a certain extent on the premise of ensuring the decoding quality. Because the positive and negative wavelet coefficients are coded separately, the amount of calculation is increased, resulting in the decrease of coding speed, but it is within the acceptable range.

\section{EXPERIMENT TEST AND RESUlT ANALYSIS}

The wavelet image generated from wavelet transform has the equal data to that of the original image, suggesting that wavelet transform itself doesn't have the function of compression. The reason why it is used in image compression is that the wavelet image it generates has different characteristics from the original image, as shown by: the image energy is mainly distributed in the low-frequency part with much less in the horizontal, vertical and diagonal parts; and the horizontal, vertical and diagonal parts represent the edge information of the corresponding parts and they have obvious direction. Low-frequency part can also be called as bright image while the horizontal, vertical and diagonal parts as the detail images. This paper selects the standard test images Cameraman and Tire for experiments to compare the performance of the proposed algorithm in this paper, the cross trace algorithm and wavelet-similarity ratio algorithm and get the PSNR value and the running time (as shown in Table 1 and Table 2) in different neighborhood $K$.

Table 1. Experiment comparison result (Image Cameraman)

\begin{tabular}{|c|c|c|c|c|c|c|}
\hline \multirow{2}{*}{$\begin{array}{c}\text { Value } \\
\text { of } k\end{array}$} & \multicolumn{2}{|c|}{$\begin{array}{c}\text { Cross trace } \\
\text { algorithm }\end{array}$} & \multicolumn{2}{|c|}{$\begin{array}{c}\text { Wavelet-similarity } \\
\text { ratio algorithm }\end{array}$} & \multicolumn{2}{c|}{ Proposed algorithm } \\
\cline { 2 - 7 } & $\begin{array}{c}\text { Time } \\
(\mathrm{s})\end{array}$ & $\begin{array}{c}\text { PSNR } \\
(\mathrm{dB})\end{array}$ & $\begin{array}{c}\text { Time } \\
(\mathrm{s})\end{array}$ & $\begin{array}{c}\text { PSNR } \\
(\mathrm{dB})\end{array}$ & $\begin{array}{c}\text { Time } \\
(\mathrm{s})\end{array}$ & $\begin{array}{c}\text { PSNR } \\
(\mathrm{dB})\end{array}$ \\
\hline
\end{tabular}




\begin{tabular}{|c|c|c|c|c|c|c|}
\hline 0 & 0.72 & 28.46 & 0.9 & 32.05 & 0.86 & 32.89 \\
\hline 1 & 0.77 & 29.57 & 0.95 & 32.13 & 0.82 & 32.57 \\
\hline 2 & 0.98 & 29.73 & 1.07 & 32.44 & 1.03 & 32.66 \\
\hline 3 & 1.1 & 29.82 & 1.13 & 32.78 & 1.16 & 33.12 \\
\hline 4 & 1.26 & 30.29 & 1.19 & 32.72 & 1.28 & 33.35 \\
\hline 5 & 1.41 & 30.13 & 1.28 & 33.05 & 1.39 & 33.48 \\
\hline 10 & 2.15 & 31.48 & 1.72 & 33.53 & 2.05 & 33.94 \\
\hline 15 & 2.82 & 32.56 & 2.25 & 33.62 & 2.74 & 34.51 \\
\hline 20 & 3.39 & 32.97 & 2.57 & 33.9 & 3.23 & 34.84 \\
\hline 30 & 4.53 & 33.58 & 3.25 & 34.11 & 4.17 & 34.83 \\
\hline
\end{tabular}

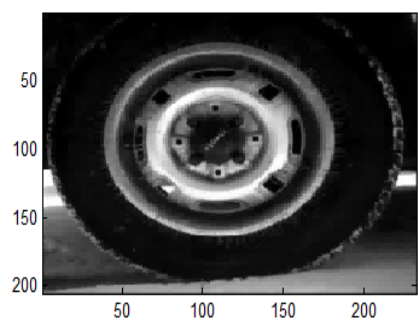

(c) Compressed image

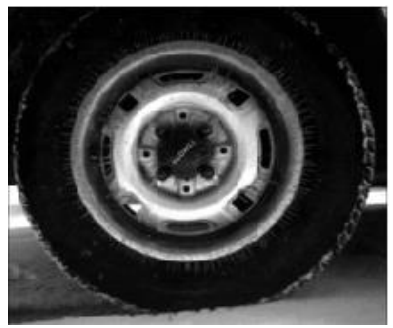

( d ) Reconstructed image

Table 2. Experiment comparison result (Image Tire)

\begin{tabular}{|c|c|c|c|c|c|c|}
\hline \multirow{2}{*}{$\begin{array}{c}\text { Value } \\
\text { of } k\end{array}$} & \multicolumn{2}{|c|}{$\begin{array}{c}\text { Cross trace } \\
\text { algorithm }\end{array}$} & \multicolumn{2}{c|}{$\begin{array}{c}\text { Wavelet-similarity } \\
\text { ratio algorithm }\end{array}$} & \multicolumn{2}{c|}{ Proposed algorithm } \\
\cline { 2 - 7 } & $\begin{array}{c}\text { Time } \\
(\mathrm{s})\end{array}$ & $\begin{array}{c}\text { PSNR } \\
(\mathrm{dB})\end{array}$ & $\begin{array}{c}\text { Time } \\
(\mathrm{s})\end{array}$ & $\begin{array}{c}\text { PSNR } \\
(\mathrm{dB})\end{array}$ & $\begin{array}{c}\text { Time } \\
(\mathrm{s})\end{array}$ & $\begin{array}{c}\text { PSNR } \\
(\mathrm{dB})\end{array}$ \\
\hline 0 & 0.65 & 33.67 & 0.77 & 36.2 & 0.69 & 36.58 \\
\hline 1 & 0.71 & 33.94 & 0.85 & 36.43 & 0.74 & 37.17 \\
\hline 2 & 0.8 & 34.83 & 0.88 & 36.74 & 0.81 & 37.62 \\
\hline 3 & 0.89 & 34.72 & 0.93 & 36.86 & 0.92 & 37.85 \\
\hline 4 & 1.1 & 34.88 & 1.02 & 36.92 & 0.96 & 38.13 \\
\hline 5 & 1.23 & 34.77 & 1.3 & 36.95 & 1.18 & 38.46 \\
\hline 10 & 1.69 & 35.85 & 1.41 & 37.63 & 1.35 & 38.82 \\
\hline 15 & 2.25 & 35.76 & 1.55 & 38.08 & 1.64 & 39.17 \\
\hline 20 & 2.58 & 36.38 & 1.72 & 38.43 & 1.89 & 39.55 \\
\hline 30 & 3.21 & 37.59 & 1.96 & 38.65 & 2.37 & 39.88 \\
\hline
\end{tabular}

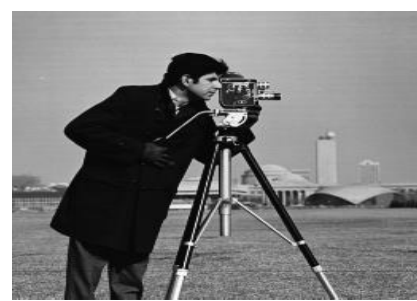

(a) Original image

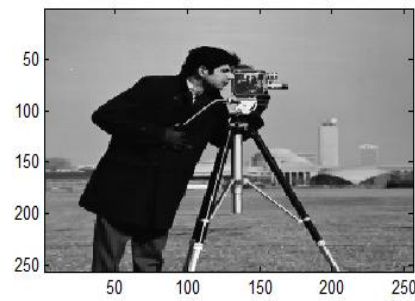

(c) Compressed image

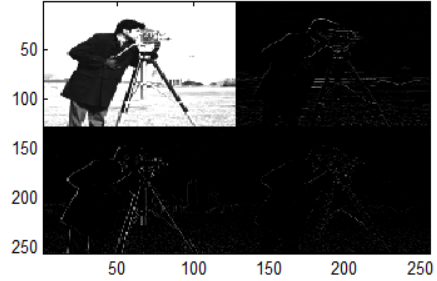

(b) Wavelet decomposition

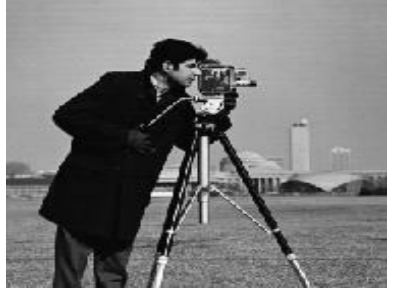

( d ) Reconstructed image

Fig 3. Image compression and reconstruction based on this algorithm ( Image Cameraman )

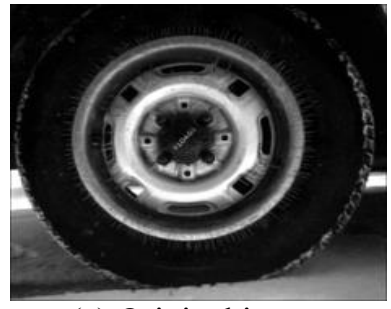

(a) Original image

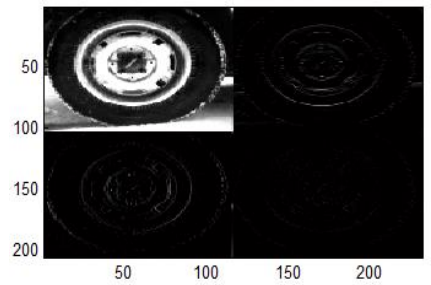

(b) Wavelet decomposition

Fig 4. Image compression and reconstruction based on this algorithm ( Image Tire )

As learnt from Table 1 and Table 2, in the same neighborhood value $k$ of the same image, the proposed algorithm in this paper takes almost the same time as cross trace algorithm, but the proposed algorithm has a higher PSNR than cross trace algorithm. In the analysis of Image Cameraman, cross trace algorithm has PSNR $=33.58$ and $t=4.53$ when $k=30$, but when $k=5$, the proposed algorithm has its PSNR reach 33.48 with only 1.39 seconds. In the analysis of Image Tire, cross trace algorithm has PSNR $=37.59$ and $t=3.21$ when $k=30$, but when $k=3$, the proposed algorithm has its PSNR reach 37.85 with only 0.92 seconds. When taking the same time, the proposed algorithm obviously has a higher PSNR than the wavelet-similarity ratio algorithm and when getting the same PSNR, the proposed algorithms takes less time, which means that the algorithm in this paper is better. Below is the wavelet analysis and reconstructed compression by the algorithm in this paper.

It can be known from the above experimental results, the algorithm makes full use of these characteristics and achieves good results. In this paper, the positive and negative coefficients are separated, and the symbols of wavelet coefficients are not recorded. At the same time, the coefficients of blocks with large standard deviation that are not suitable for non-search fractal coding are directly recorded to ensure high decoding quality. Firstly, the commonly used wavelet in image processing is used to process the image by wavelet transform, and then a positive wavelet coefficient matrix and a negative wavelet coefficient matrix are obtained according to the wavelet coefficient symbol. The two matrices are coded respectively by combining non search fractal coding technology and fractal code prediction technology. This algorithm uses the similarity between wavelet coefficients in the same direction and uses the affine transform method to obtain the wavelet coefficients with low decomposition levels from the wavelet coefficients with high decomposition levels. The lower wavelet coefficients reflect the high-frequency details of the image, and most of this part of the data is close to zero, occupying only a little energy. Even if there is a certain error, it can't be observed by the human eye.

\section{CONCLUSION AND FUTURE WORK}

This paper has combined wavelet analysis with fractal image compression coding. Through the pre-processing of wavelet transform, it has converted an image into 
low-frequency sub-band and high-frequency sub-band and then it has conduct block forking as well as fractal image compression and reconstruction on low-frequency sub-band. By use of the sparsity of fractal differential image in the low-frequency sub-band and in combination of the sparsity of high-frequency sub-band, it has performed coding reconstruction through compressed sensing and reconstruction algorithm to make up for the details of the reconstructed image. Meanwhile, based on the fact that conventional basic fractal coding uses full search, it has used the geometric features of image sub-blocks into 3 classes, restricted the search scope in the neighborhood with close feature value of the sub-blocks with the corresponding features in each class, and performed the neighborhood search for the best-matched block so as to reduce the objects to be searched and the coding time and accelerate the process while ensuring image quality. Through comparative simulation experiment, the result has shown that compared with other algorithms, the proposed algorithm in this paper has significantly shortened the time spent on image coding and greatly improved the coding speed while ensuring the quality of reconstructed image.

The limitations of this study and future directions: Fractal and wavelet theory have very good application prospects in the field of image compression, but there are still many problems, resulting in the algorithm based on this theory has not been widely used. At present, the following aspects need to be further studied. Considering the division of image blocks according to the image content, higher compression ratio and decoding quality are realized; Although the coding speed of this algorithm has increased, there will still be serious block effect at high compression ratio, and there is still a big gap in visual effect and robustness compared with other mature algorithms; This paper comprehensively analyzes various current graphics compression algorithms, tries to combine various theories, and puts forward a new image compression algorithm; This paper only studies the region of interest compression method of still image, which is not suitable for video compression. In the next step, consider the video region of interest compression technology. • •

\section{ACKNOWLEDGMENT}

This work was supported in part by the Foundation of the Shaanxi Key Laboratory of Intelligent Processing for Big Energy Data(Grant No.IPBED17), Doctor Foundation of Yan'an University (Grant No. YDBK2019-21), Nature Foundation of Yan'an University of Yan'an University, China (Grant No. YDY2019-19), and Postgraduate Teaching Reform Project of Yan'an University, China (Grant No.YDYJG2019019).

\section{REFERENCES}

[1] Halawani, Yasmin; Mohammad, Baker; Al-Qutayri, Mahmoud, "Memristor-Based Hardware Accelerator for Image Compression", IEEE Transactions on Very Large Scale Integration (Vlsi) Systems, vol. 26, no.12, pp.2749-2758, 2018.

[2] Gupta, Richa; Mehrotra, Deepti; Tyagi, Rajesh Kumar, "Comparative Analysis of Edge-Based Fractal Image Compression Using Nearest Neighbor Technique in Various Frequency Domains", Alexandria Engineering Journal, vol.57, no.3, pp.1525-1533, 2018.

[3] Uthayakumar, J.; Elhoseny, Mohamed; Shankar, K. , "Highly Reliable and Low-Complexity Image Compression Scheme Using Neighborhood
Correlation Sequence Algorithm in WSN", IEEE Transactions on Reliability, vol.69, no.4, pp.1398-1423, 2020.

[4] Chiranjeevi, Karri; Jena, Uma Ranjan, "Image Compression Based on Vector Quantization Using Cuckoo Search Optimization Technique", Ain Shams Engineering Journal, vol.9, no.4, pp.1417-1431, 2018.

[5] Luesi, Iiris; Bolotnikova, Anastasia; Daneshmand, Morteza, "Optimal Image Compression Via Block-Based Adaptive Colour Reduction With Minimal Contour Effect", Multimedia Tools And Applications, vol. 77, no. 23, pp.30939-30968, 2018.

[6] Alkishriwo, Osama A. S. , "Image Compression Using Adaptive Multiresolution Image Decomposition Algorithm", IET Image Processing, vol.14, no.14, pp.3572-3578, 2020.

[7] Wang J, Zheng N. "A Novel Fractal Image Compression Scheme With Block Classification and Sorting Based on Pearson's Correlation Coefficient", IEEE Transactions on Image Processing, vol. 22, no.9, pp.3690-3702, 2013.

[8] Valsesia, Diego; Magli, Enrico, "High-Throughput Onboard Hyperspectral Image Compression With Ground-Based CNN Reconstruction", IEEE Transactions on Geoscience and Remote Sensing, vol. 57, no. 12, pp. 9544-9553, 2019.

[9] Ganasala, Padma; Prasad, Achanta Durga, "Medical Image Fusion Based on Laws of Texture Energy Measures in Stationary Wavelet Transform Domain", International Journal of Imaging Systems and Technology, vol.30, no.3, pp.544-557, 2020

[10] Bozhokin, S. V.; Sokolov, I. M. , "Comparison of the Wavelet and Gabor Transforms in the Spectral Analysis of Nonstationary Signals", Technical Physics, vol. 63, no. 12, pp. 1711-1717, 2018.

[11] Ayalapogu, Ratna Raju; Pabboju, Suresh; Ramisetty, Rajeswara Rao, "Analysis of Dual Tree M-Band Wavelet Transform Based Features for Brain Image Classification", Magnetic Resonance in Medicine, vol. 80, no. 6, pp. 2393-2401, 2018.

[12] Mendhe P, Balpande R, Khobragade A. "Fast fractal image encoding scheme based on Absolute value of Pearson correlation coefficient", International Conference on Communications and Signal Processing (ICCSP).pp.1036-1040, 2015.

[13] Taheri, Amir Masoud; Mahdavi-Nasab, Homayoun, "Sparse Representation Based Facial Image Compression Via Multiple Dictionaries and Separated ROI", Multimedia Tools and Applications, vol. 77, no.23, pp.31095-31114, 2018.

[14] Naseer, Yasir; Shah, Tariq; Attaullah, "Advance Image Encryption Technique Utilizing Compression, Dynamical System and S-Boxes", Mathematics and Computers in Simulation, vol.178, pp. 207-217, 2020.

[15] Krishnaraj, N.; Elhoseny, Mohamed; Thenmozhi, M, "Deep learning model for real-time image compression in Internet of Underwater Things (IoUT)", Journal of Real-Time Image Processing, vol.17, no.6, pp.2097-2111, 2020.

[16] Zhao DY, Zhu SP, Wang FC. "Lossy Hyperspectral Image Compression Based on Intra-Band Prediction Andinter-Band Fractal Encoding", Computers \& Electrical Engineering, vol.54, pp.494-505, 2015.

[17] Yuan, Xin; Haimi-Cohen, Raziel, "Image Compression Based on Compressive Sensing: End-to-End Comparison With JPEG", IEEE Transactions on Multimedia, vol. 22, no.11, pp. 2889-2904, 2020.

[18] Cai, Chunlei; Chen, Li; Zhang, Xiaoyun, "Efficient Variable Rate Image Compression With Multi-Scale Decomposition Network", IEEE Transactions on Circuits and Systems for Video Technology, vol29, no. 12, pp. 3687-3700, 2019.

[19] Wang XY, Zhou DD, Wang N. "Fractal Image Coding Algorithm Using Particle Swarmoptimisation and Hybrid Quadtree Partition Scheme", IET Image Processing, vol.9, no.2, pp.153-161, 2015.

[20] Lv, Xiupin; Liao, Xiaofeng; Yang, Bo, "A Novel Scheme for Simultaneous Image Compression and Encryption Based on Wavelet Packet Transform and Multi-Chaotic Systems", Multimedia Tools and Applications, vol.77, no.21, pp.28633-28663, 2018.

[21] Hurtik, Petr; Tomasiello, Stefania, "A Review on the Application of Fuzzy Transform in Data and Image Compression", Soft Computing, vol.23, no.23, pp.12641-12653, 2019

[22] Bhattacharya N, Roy SK, Nandi U, et al. "Fractal Image Compression Using Hierarchical classification of Sub-Images", Proceedings of the 10th International Conference on Computer Vision Theory and Applications, pp.46-53, 2015.

[23] Balakrishnan, Binu; Darsana, S. H.; Mathews, Jayamol, "Satellite/Aerial Image Compression Using Adaptive Block Truncation Coding Technique", Journal of the Indian Society of Remote Sensing, vol.46, no.11, pp. 1761-1771, 2018. 
[24] Huang, Hsin-Hsiung; Girimurugan, Senthil B. , "A Novel Real-Time Genome Comparison Method Using Discrete Wavelet Transform", Journal of Computational Biology, vol.25, no.4, pp. 405-416, 2018.

[25] Yeap, Yew Ming; Geddada, Nagesh; Ukil, Abhisek, "Analysis and Validation of Wavelet Transform Based DC Fault Detection in HVDC System", Applied Soft Computing, vol.61, pp.17-29, 2017.

\section{Creative Commons Attribution License 4.0 (Attribution 4.0 International, CC BY 4.0)}

This article is published under the terms of the Creative Commons Attribution License 4.0

https://creativecommons.org/licenses/by/4.0/deed.en_US 